\title{
Age-related slowing of movement as basal ganglia dysfunction
}

\author{
L. L. Saling • J. G. Phillips
}

Received: 29 November 2007 / Accepted: 22 July 2008 / Published online: 12 August 2008

(C) EGREPA 2008

\begin{abstract}
Attributions of age-related deficits in motor function to structural changes are compromised once the elderly exhibit lower error rates. This is because performance decrements observed in older adults are attributed to inferred strategic preferences for accuracy over speed. To understand genuine age differences in performance, we argue in the following theoretical paper that research needs to resolve methodological shortcomings and account for them within theoretical models of aging. Accounts of aging need to directly manipulate or control strategic differences in performance while assessing structural deficits. When this is done, age-related changes in motor control resemble the intermittencies of control seen in basal ganglia disorders. Given homologous circuitry in the basal ganglia, such observations could generalize to age-related changes in cognitive and emotional processes.
\end{abstract}

Keywords Aging · Slowing of performance · Strategy ·

Basal ganglia $\cdot$ Performance operating characteristics

Psychomotor function is a major contributor to the independence of older adults. Unfortunately, accounts of psychomotor change with age are complicated by difficulties in unequivocally addressing age-related change. Although gerontologists may wish to identify real structural deficits that can account for the behavior of older adults, the ability to address these deficits is limited by the ability to draw inferences from

L. L. Saling $(\bowtie)$

School of Humanities and Social Sciences, Charles Sturt University,

Wagga Wagga, New South Wales 2678, Australia

e-mail: 1saling@csu.edu.au

J. G. Phillips

Department of Psychology, Monash University,

Clayton, Victoria 3800, Australia observable behaviors in the light of potential strategic variation [13, 14, 15, 19, 31, 75]. By structural changes, we mean physiological changes associated with brain structures, in this case, the basal ganglia and its associated functional circuitry (e.g., see [39], for a discussion of structural and functional basal ganglia changes associated with aging).

Such difficulties then cause problems in the tractability of models of age-related deficits, and even when specific behaviors are readily observable, there may be problems of generalizability when extrapolating to other domains.

Models are possible, but there are problems of testability. This is because performance differences may simply reflect a strategic preference for accuracy over speed. As there is a tendency for differences in strategy to be inferred from performance decrements, this undermines any attributions of age-related changes in performance to structural changes. For there to be progress in the understanding of genuine age differences in performance, accounts of aging need to address strategic differences in performance while assessing structural deficits. This theoretical paper therefore seeks to address these problems by incorporating strategic variation into accounts of age-related changes in performance, delineating means whereby this can be addressed theoretically. By means of a discussion of empirical research, this paper demonstrates how strategic differences can be managed through the use of performance operating characteristics. Such solutions are therefore tractable and testable. Studies that control strategy reveal that the psychomotor decline associated with aging exhibits similarities to the failures of automaticity seen in basal ganglia disorders.

As will be explained, although these issues are readily addressed in the domain of movement, the motor circuits of the basal ganglia are homologous to the circuits associated with cognition and emotion [76], and so an understanding 
of aged motor function has the potential to generalize to other domains. This implies that similar mechanisms could apply for other processes, which may be compromised in aging such as cognition or emotion.

\section{Strategic differences between older and young adults?}

Researchers studying age-related differences in performance have suggested that slowing of the speed of performance of older adults, when compared to the performance of young adults, is in part due to a change in performance strategy [13, 14, 15, 19, 63]. In particular, these authors have postulated that older adults select a strategy in which greater emphasis is placed on response accuracy, with a resulting decrement in speed of performance.

Differences noted in the performance of young and older adults are therefore often considered to reflect a preference for caution over speed. Unfortunately, such an approach is ad hoc. Once the data is collected, differences between young and older adults can then be assigned a hypothetical value for a speed accuracy trade off (i.e., slow and cautious versus fast and careless). Only if accuracy is documented and the elderly are slow and inaccurate and the young are fast and accurate can a difference in processing ability be firmly announced [2]. Otherwise, strategic differences obscure interpretations of data, as age-related differences in performance can be dismissed as mere strategic variation. This methodological issue hinders the development of accounts of the aging process.

\section{Adaptive control models}

There are classes of simple models available that can incorporate strategic differences within a context of reduced functional capacity (e.g., [73]). Such models make predictions as to performance at a specific level of caution and level of difficulty [73]. These adaptive information processing models act to regulate their own performance to maintain a controlled quantity (e.g., confidence and accuracy). For instance, where such models detect poor confidence (indicated by poorer balance of evidence for a decision), the processing criteria is adjusted upwards so that more information is processed for subsequent decisions.

Such models can address strategic differences. For instance, predictions can be made as to behavior on the basis of high or low caution [73]. Hence, such models have within the context of discussions of strategic differences and age. For instance, confidence covaries with error rates in older drivers [49] however; models invoking adaptive control suffer from problems of testability. Confidence can be a difficult variable to measure. And different adaptive models can be very similar in their performance (e.g., [73]), the issue here being the number of hypothetical variables in relation to the number of observable variables [6]. For such reasons, it can be better to address age-related changes in circumstances where performance can be directly measured and documented.

\section{Performance operating characteristics}

If there is a strategic preference for accuracy over speed of response with advancing age, it can be controlled. A performance operating characteristic (POC) is one means of managing this. POC methodology involves the sampling of performance under several instructional sets (e.g., instructions for speed or accuracy). Speed of performance is then plotted against accuracy (see [2]). Through explicit manipulation of instructions or payoffs, one can produce POCs enabling a determination of performance capabilities over various experimental conditions [48]. POC methodology incorporates the assumption that human information processing has a limited capacity and that multiple processes must compete for limited resources [26]. Thus, in the context of dual task performance, such that multiple tasks are competing for limited resources, the resources required by each task can be calculated. This method offers a better way of exploring age-related performance changes.

\section{Response preparation}

For example, the following study controls the confounding issue of strategy when addressing age-related differences in response preparation. Response planning is a feedforward process. Therefore, if there is no plan for movement, then people need to stop and check and engage in feedback guided control.

Hitherto, interpretations of preparatory effects had been compromised by ad hoc reference to strategic differences. Although elderly reaction times are slower, they are sometimes not specifically linked to movement parameters, the suggestion being that the elderly are just less willing to prepare their responses, preferring instead to guide movements during execution [3]. Bellgrove et al. [7] investigated whether older adults were less efficient at programming their movements or just had a strategic preference for online guidance. To determine this, Bellgrove et al. [7] controlled the degree to which older and young adults could program their movements. As such, in one condition, a cue was provided as to target location (facilitating response preparation), while in another condition, no cue was provided. When programming of movements was restricted, such that both groups were limited to online guidance, 
the movements of older adults were still less efficient than those of young adults. Older adults had difficulties associated with response preparation, programming, and reprogramming.

\section{Response execution}

The finding that older adults have difficulty associated with response preparation, programming, and reprogramming demonstrates that older adults have genuine deficits. Response execution is impaired when programming is restricted, thus indicating that slowing of performance in older adults is not merely due to a strategic preference for online guidance of performance. When movement speed is controlled, older adults still have difficulties associated with trajectory formation (see [47]).

Unfortunately, age-related differences in response execution are also associated with suggestions that older adults prefer accuracy to speed $[13,14,15,19,31,75]$. Hence, agerelated differences could merely reflect a preference for accurate movements at the expense of speed. As indicated previously, such differences in strategy, when inferred from the data, can be considered to be ad hoc when explaining age-related changes in response execution. As such, in order to verify the hypothesis that the performance changes observed in older adults reflect a change in performance strategy [13, 14, 15, 19, 31, 63], Morgan et al. [47] explicitly manipulated performance variables.

Incorporating the logic of POC methodology to control for strategic differences in the performance of elderly participants, Morgan et al. [47] dictated the strategy utilized in performance. Morgan et al. [47] trained older adults to perform at the preferred speed of young adults and found that the performance of older adults was more hesitant and involved a greater number of submovements than that of young adults. The authors concluded that the performance of older adults is actually characterized by motor incoordination as opposed to merely representing strategic differences in the form of a preference for slower movement. Hence, there are differences in trajectory formation.

To understand trajectory control in the context of the drawing movements of older adults, Saling and Phillips [60] explicitly manipulated performance variables. In particular, Saling and Phillips [60] investigated the operation of the one-third power law, which describes the relationship between geometric properties of a trajectory (radius of curvature) and movement kinematics (tangential/ angular velocity). Saling and Phillips [60] compared the drawing movements of young and older adults as a function of a central mechanism (timing cue) and a peripheral mechanism (joint combinations). Central and peripheral variables were explicitly manipulated.
Saling and Phillips [60] dictated the speed of movement performance in order that strategic differences would not confound the interpretation of performance. The power law broke down in older adults under conditions of mechanical limitation, suggesting the presence of age-related degeneration of peripheral mechanisms. Since the power law breaks down in older adults under conditions of biomechanical constraint, it provides further possible measures for quantifying some age-related motor changes. This suggests that the joint combinations used in movement tasks may underpin some performance changes [61]. Therefore, through the explicit manipulation of performance variables, including speed of performance and joints used in movement completion, a determination was undertaken as to the possible mechanisms underlying age-related slowing. Furthermore, kinematic analysis was also undertaken in this study, which demonstrated that the movements of older adults are less smooth and are characterized by a greater number of submovements than those of young adults, even when speed is controlled.

In general terms, kinematic analysis of the movements of older and young adults has revealed a greater number of submovements associated with the performance of older adults as compared to young adults [47, 60]. The performance of older adults is generally poor and hesitant, resembling to some degree the motor behavior of patients with movement disorders [38]. Instead of a preference for accuracy, we see an intermittency of control associated with the movements of the elderly. This does not seem to be directly related to speed of output as even when controlling speed older adults perform a greater number of submovements than do young adults at the same speed [47, 60]. Therefore, irrespective of whether there is a problem associated with force production or the monitoring of output, older adults engage in more regular "stops" to check, amend, or update their performance. Note that these are not hypothesized stops but actual intermittencies measured in movement trajectories.

\section{Medical models of age-related slowing}

In particular, the movements of older adults have some elements in common with the movements of patients with Parkinson's disease. The movements of older adults are slower, and there are discontinuities in their trajectories [38]. Furthermore, irregularities in the accelerative phase has been observed in both older adults [47] and patients with Parkinson's disease [51], even when allowing for differences in movement duration.

Parkinson's disease results from a loss of dopaminergic cells in the substantia nigra (for a review, see [76]), leading to a decrease in levels of brain dopamine. Since these 
neurons then project to the striatum, there is ultimately a disruption of both the direct and indirect pathways within the basal ganglia. Parkinson's disease is characterized by a number of symptoms including resting tremor and rigidity as well as difficulty in the initiation (akinesia) and execution (bradykinesia) of movement [41, 42, 51].

The symptoms associated with Parkinson's disease arise when approximately $80 \%$ of dopaminergic neurons in the substantia nigra projecting to the striatum are lost [35]. The symptoms of Parkinson's disease are associated with a loss of innervation to the basal ganglia [42]. As the basal ganglia regulate the activity of key cortical areas, there are liable to be disturbances to cortical activity as well [21].

Parkinson's disease selectively targets particular cell types, and hence, the neuropathological process associated with Parkinson's disease can occur wherever these cells are present [29]. Thus, for example, some regions of the allocortex, the neocortex, and the mesocortex are vulnerable to the neuropathological process underlying Parkinson's disease [11]. In particular, the neuropathology of Parkinson's disease consists of spindle or thread-like Lewy neurites within the somata of vulnerable neurons [10].

A number of parallels have been drawn between Parkinson's disease and normal aging. Indeed, changes that are characteristic of Parkinson's disease are common in elderly populations [11].

Firstly, the slowing of speed of processing associated with aging is analogous to the slowing of movement execution that is observed in Parkinson's disease [38]. Secondly, there is evidence that there is a decrease in levels of brain dopamine in the elderly [5] leading to the assertion that age-related motor changes may reflect insufficient dopamine. In particular, a decrease in nigostriatal dopaminergic innervation has been noted in both normal aging and Parkinson's disease [9]. This decline in dopamine levels is associated with motor changes in older adults even in the absence of a diagnosis of Parkinson's disease [74].

In light of these similarities, Parkinson's disease has been offered as a potential model of age-related slowing of the motor system [38]. Although other disease entities, including cerebellar dysfunction, may also provide models of accelerated aging, we will focus on Parkinson's disease. This is because Parkinson's disease is currently conceptualized as the prototypical motor disorder and hence provides an opportunity for comparison with age-associated motor deficits. Furthermore, the symptoms associated with Parkinson's disease including the slowing of motor processes as well as the hesitancy associated with motor production is comparable to the motor behavior of older adults.

Cerebellar dysfunction could also be offered as a model of age-related decline in motor function, but clear specific models are simply less available. There are few conditions that specifically target the cerebellum in humans (e.g.
Friedrich's ataxia and cerebellar atrophy), and they are comparatively rare [20]. The cerebellum is of course vulnerable to a variety of non-specific conditions and lesions [22], but such research then tends to study symptoms of cerebellar dysfunction rather than specific localized instances of cerebellar damage. For instance, symptoms of cerebellar dysfunction tend to be observed clinically in patients with essential tremor or conditions, such as multiple sclerosis, and cerebellar dysfunction can be observed as a function during alcohol intoxication or abuse [22]. Nevertheless, such conditions also involve damage to other structures, and as such, we argue that there are fewer clear clinical models of cerebellar damage to work with.

The mechanisms that are thought to underlie the motor losses of Parkinson's disease may also explain age-related slowing. Drawing analogies between disease processes and age-related changes offers possible therapies for age-related motor changes as Parkinson's disease could provide a testbed for interventions, including the use of dopamine agonists, which may serve to ameliorate age-related motor deficits.

Thus, although age-related slowing has been characterized as a strategic preference for online control rather than preparation or accuracy over speed, instead it appears that the movements of older adults are excessively hesitant resembling movement observed in disease processes [38]. As such, in aging as in Parkinson's disease, there is the suggestion of basal ganglia dysfunction as the neural substrate underlying slowing of performance (e.g., see [59] for a discussion of age-related changes as basal ganglia dysfunction). As will be outlined, it appears that lower level (subcortical) functions are compromised and hence are less reliable. Therefore, the burden of control of performance is increasingly shifted to other (higher level) systems. Thus, the computational burden on the system is increased, and performance is compromised due to limited capacity. There are changes in the pattern of brain activation with the development of skill. Wu et al. [79] demonstrate that, as skill develops, there is a reduction in overall brain activity, with retention in activity in a limited number of structures. It thus appears that solutions to problems have been established and that they involve basal ganglia activity. Nevertheless, with age [78] or Parkinson's disease [77], these previously established solutions to problems no longer seem to be available for routing through the basal ganglia.

\section{Age-related motor changes: a failure of automatic processes?}

The functional loss associated with aging and Parkinson's disease appears to be a loss of automaticity. Hence, the nature of automatic processes will be outlined. The 
acquisition of automaticity appears to be linked to a reduction in global brain activation and a shift from cortical structures to subcortical structures, in particular the basal ganglia [62]. Indeed, patients with disorders of the basal ganglia (such as Parkinson's disease) exhibit slowing of the initiation and execution of behavior (motor losses) that appear to result from a dramatic failure in the acquired forms of automatic behavior [52, 77]. As we have indicated, there appears to be an analogous loss of automatic processes with advancing age. Indeed, $\mathrm{Wu}$ and Hallett [78] noted that older adults required more training than young adults in order to achieve automaticity of performance. Furthermore, there was greater brain activation associated with automatic processing in the elderly than in young adults. In particular, there was greater activation of the bilateral anterior cerebellum, premotor area, parietal cortex, left prefrontal cortex, anterior cingulate, caudate nucleus, and thalamus in older adults.

This increase in activation is of great interest, as automaticity typically is associated with a reduction in brain activity [62]. This suggests that older adults are recruiting additional structures (and hence relying on controlled processes) to compensate for the degradation of automatic processes. This greater brain activity adds to the computational burden and taxes limited processing resources.

There are of course a variety of mathematical models purporting to describe basal ganglia function [4, 28]. Such models, however, represent descriptions based upon a level of knowledge or understanding of neuroanatomy or physiology. Imaging studies provide evidence of the involvement of specific structures, albeit without necessarily determining the time course or the degree of connectivity of the structures. As such, we highlight relevant imaging studies that indicate functional reorganization associated with aging.

Indeed, functional imaging studies indicate that there is greater activation of motor cortices associated with aging (e.g., [36]). This increase in activation is likely to be the result of compensation for the degeneration of subcortical circuits possibly representing functional reorganization of motor loops [70]. As such, in older adults, there is recruitment of additional brain areas to compensate for the age-related decline associated with brain function [32, 33]. As such, the task is still performed but less efficiently than in younger adults.

Hence, slowing of speed of processing with age does not appear to represent a preference for slower, more accurate movements, or reluctance to plan movements, but rather a genuine deficit. A failure of automaticity in older adults could account for age-related slowing.

Although a failure of automatic processes has the potential to serve as an explanation for age-related motor changes, automaticity itself is a difficult construct to measure [62]. The classical distinction between automatic and controlled processes relies on the defining features of intention, attention, and awareness (see [40]), although the construct has evolved significantly since its inception. Automatic processes are typically conceptualized as fast, obligatory (stimulus-driven), and lacking awareness, whereas controlled processes are conceptualized as slow, intentional, and aware [40].

Although the dominant account of automaticity dichotomizes automatic and controlled processes on the basis of the presence or absence of the features of intention, attention, and awareness, this distinction is not borne out empirically. Automatic processes can be influenced by intention, attention, and awareness and can be modified in a contextually appropriate fashion [54, 62]

Automaticity, or its absence, has often been inferred from task performance rather than being subjected to empirical validation. In particular, automaticity, or its absence, is often inferred from the speed of task performance [34]. There is thus a need to search for directly observable instances of automatic behaviors to supply some converging lines of evidence as to the validity of this position. Fortunately, an examination of postural reflexes in the elderly provides an opportunity for empirically validating the theory that age-related slowing derives from the degradation of automatic processes.

\section{Deficits in postural reflexes in aging}

Reflexes are considered to be prototypical automatic movements. Maintenance of an upright posture is considered to be an automatic activity [44]. Postural reflexes also facilitate the transition from posture to movement. There are two primary types of postural reflex activity: anticipatory and reactive. Anticipatory or feedforward processes compensate for the destabilization resulting from voluntary movement, whereas reactive or feedback activity occurs in response to changes in the environment that require postural stabilization [25]. Changes in postural reflexes associated with advancing age provide an opportunity to explore the operation of automaticity in older adults.

Feedforward postural adjustments refer to preparatory postural adjustments, while feedback control refers either to reflexive corrections or voluntary stabilizing movements in response to postural perturbations [68].

Deficits in postural reflexes have been observed in the elderly [27] and in patients with Parkinson's disease [18]. Postural responses tend to be slower and less reliable with age. Such deficits result in difficulties associated with balance and postural adjustments for movement [27]. Typically, it is the more reactive/feedback postural activity that degrades with advancing age [34, 72] and in patients with Parkinson's disease [8]. The basal ganglia have been implicated in this unconscious postural dyscontrol. 
The postural changes noted in Parkinson's disease reflect, at least in part, an impairment associated with the ability to process proprioceptive information [43, 66, 81].

Older adults and patients with Parkinson's disease often have difficulty in responding to postural perturbations including those that are required on uneven surfaces or moving platforms and have problems integrating reflexes with ongoing activity [17, 68]. Problems associated with reflexes increase reliance on the visual frame [72], and hence, if the visual frame is not straight [50] or if they are deprived of visual feedback [8], they are more likely to fall. In this context, vision represents a supervisory control process, whereas reflexes are considered to be automatic.

As there is a greater reliance on visual feedback to maintain balance, owing to less efficient postural reflexes, balance can be seen as one area where there is a decrease in automaticity and an increased dependence on voluntary processes with advancing age [68]. Melzer et al. [45] argue that older adults are unable to automatically activate corrective strategies to maintain posture and hence must recruit alternative, less efficient, controlled processes to maintain posture. Melzer et al. [45] have demonstrated that the elderly control posture by means of a process of central reorganization, requiring cognitive processing, to compensate for impaired postural corrective processes. Such corrective responses may be slower [68].

A consideration of postural reflexes thus reifies the shifting interplay between controlled and automatic processes with age. The deficits noted in postural reflexes associated with aging, like those associated with Parkinson's disease, can be attributed to basal ganglia dysfunction and decreased dopamine levels (see [16]) and hence provide one means of empirically validating the failure of automatic processes in elderly populations and the greater reliance on controlled processes. A failure of automaticity appears to represent one of the mechanisms underpinning age-associated motor slowing, and there are structural correlates in this case, such as basal ganglia dysfunction that may account for these problems. The joints used in movement completion also contribute to such changes, suggesting that biomechanical mechanisms are, in part, responsible for age-related performance decrements [60].

The movements of older adults are associated with a greater number of submovements and are more hesitant resembling, to some degree, the movements associated with disease processes and possibly reflecting a shift toward controlled mechanisms, as automatic mechanisms become less reliable. Since automatic processes are thought to be compromised in older adults, this has implications for the operation of controlled processes. Automatic and controlled processes involve different brain structures and mechanisms [62]. The transition from controlled to automatic processing appears to involve the basal ganglia, but some involvement of cortical structures remains such that automatic processes are able to be monitored and modified in a contextually appropriate fashion [62].

There are multiple levels involved in motor control, whereby higher order processes may delegate to the lower order processes, thus reducing the computational burden on the system. In this case, the lower order automatic processes are compromised, and as such, a burden is imposed on controlled processes. The controlled process is less efficient and represents a more indirect solution. Thus, although a particular task may still get done, it is achieved at a cost. And as explained in this paper, this is not merely inferred but directly observed and documented by means of an examination of postural reflexes.

\section{Generalizability to other domains}

We suggest that what is discovered in the area of motor function, where behavior can be more readily observed and documented, may apply for other functions as well. Afferents to the basal ganglia have a topographic organization that tends to maintain the topology of the cerebral cortex [1]. For instance, the specific disturbances seen in patients with focal dystonia (disorders where specific limb segments are affected) are potentially clinical manifestations of this segregation of influences within the basal ganglia [67]. Indeed, studies of the effects of microstimulation and of single-cell activity indicate that an anatomic somatotopy of the basal ganglia is maintained at a functional level. This led Alexander et al. [1] to identify parallel functional circuits within the basal ganglia associated with motion, eye-movements, cognition, and emotion.

Wichmann and Delong [76] observe the pattern of connectivity. The basal ganglia consist of a number of parallel circuits with similar homologous connectivity. In each case, multiple cortical areas feed into the basal ganglia which then target a specific cortical structure. For example, motor cortical areas (premotor, motor cortex, and supplementary motor area) project through the putamen and then target the supplementary motor area, while cognitive cortical areas (dorsolateral, prefrontal, lateral orbitofrontal cortex) project through the caudate nucleus and target the dorsolateral cortex. Limbic cortical areas associated with emotion (anterior cingulate, medial orbitofrontal cortex) project via the ventral striatum and target the anterior cingulate [76]. Therefore, although motor behavior represents only one domain in which slowing of processing can be documented, the findings in this area can potentially be generalized to other domains including cognition and emotion.

As each of these circuits have similar connection and function, understanding the age-related changes in the motor circuit of the basal ganglia may actually shed light 
upon the age-related changes in other systems where speed can be an issue, such as information processing $[53,56]$, cognition [71], or emotion [55]. For example, Touron and Hertzog [71] have found that older adults were less able than young adults to acquire automaticity in the context of a memory task. This is thought to be because older adults are slower to shift to a memory retrieval strategy, decreasing the automaticity and hence efficiency of task performance.

Hitherto, studies of the motor functions indicate that the basal ganglia have a role in activating and regulating motor function. As the basal ganglia have a number of homologous circuitry, a regulatory role for the other circuits is implied. Hence, the basal ganglia may play a role in switching on and off functions such as emotion and cognition. There are indications to support this hypothesis. For instance, there is some indication that dopamine is involved in moderating the response to reward as well as the addictive potential of substances and behaviors (e.g., [80]). Furthermore, deficient fronto-cortical function has been implicated in depression. Other research indicates a potential role for the basal ganglia in cognition. For example, a slowing of cognitive processes is linked to a number of subcortical conditions (e.g., [46]). Hence, a study of motor function could have implications for cognition and emotion. Future research could consider depression and cognitive slowing in the elderly, although it is not clear whether it is necessarily the level of activity of brain structures or their interconnectivity that is important $[23,46]$.

At a therapeutic level, dopamine agonists have been found to be effective in the management of refractory depression [69] bipolar depressive disorders [24] as well as depression associated with Parkinson's disease [65]. Furthermore, Rammsayer and Stahl [58] found that dopamine agonists facilitated early cognitive and perceptual aspects of sensorimotor processing such that the time required for early stimulus processing was reduced.

Conversely, studies that have investigated the effect of DA antagonists on the behavior of healthy participants have noted that such agents typically impair performance. In general terms, such studies have revealed that DA antagonists decrease alertness [64], modulate the response to reward [12, 80], and impair learning [82], memory [57], and motor performance [37]. As such, alternatives to DA antagonists might be sought with advancing age.

There is some controversy in the literature as to whether automatic processes are preserved (e.g., see [30]) or reduced with advancing age. We suggest that, in part, this arises owing to the simplistic usage of the construct. This will not be elaborated upon here, but this issue is discussed at length in Saling and Phillips [62]. Suffice to say that the current dichotomous distinction made between automatic and controlled processes fails to account for the real world complexity of automatic behavior, thus we propose that, by looking at the neural basis of the behavior, one can better explain the behavior in question.

\section{Conclusions}

Psychomotor function is a major contributor to independence, but accounts of psychomotor change with age are complicated by difficulties associated with the interpretation of age-related changes. The identification of structural deficits is limited by the ability to draw inferences from observable behaviors in light of potential strategic variation $[13,14,15,19,31,63,75]$. There has been a tendency, however, for researchers to overlook this problem owing to the assumption that it cannot be overcome as it is not tractable or testable.

When strategic variation is explicitly controlled by means of generating POCs (see [2]), a consideration of psychomotor function in older adults indicates genuine deficits rather than a strategic preference. Further examination of motor function suggests failures in the automatic execution of behavior such that older adults must rely on controlled processes, thus taxing limited processing resources. Changes in motor processes with advancing age can provide markers of more general neurophysiological decline. Furthermore, the adoption of basal ganglia dysfunction as an explanation of age-related motor slowing indicates possible interventions and potentially offers a means of generalizing from documentable motor deficits to other domains such as cognition and emotion owing to homologous basal ganglia circuitry.

\section{References}

1. Alexander GE, Crutcher MD, DeLong MR (1990) Basal gangliathalamocortical circuits: Parallel substrates for motor, oculomotor, "prefrontal" and "limbic" functions. Prog Brain Res 85:119-146 doi:10.1016/S0079-6123(08)62678-3

2. Allen PA, Murphy MD, Kaufman M, Groth KE, Begovic A (2004) Age differences in central (semantic) and peripheral processing: The importance of considering both response times and errors. J Gerontol Psychol Sci 59B:210-219

3. Amrhein PC, Stelmach GE, Goggin NL (1991) Age differences in the maintenance and restructuring of movement preparation. Psychol Aging 6:451-466 doi:10.1037/0882-7974.6.3.451

4. Atallah HE, Frank MJ, O'Reilly RC (2004) Hippocampus, cortex and basal ganglia: Insights from computational models of complementary learning systems. Neurobiol Learn Mem 82:253267 doi:10.1016/j.nlm.2004.06.004

5. Backman L, Farde L (2005) The role of dopamine systems in cognitive aging. In: Cabeza R, Nyberg L, Park D (eds) Cognitive Neuroscience of aging: linking cognitive and cerebral aging. Oxford University, New York, NY, pp 58-84

6. Bamber D, van Santen J (2000) How to tell whether a model is testable: The example of discrete-state selective-influence models. J Math Psychol 44:20-40 doi:10.1006/jmps.1999.1275 
7. Bellgrove MA, Phillips JG, Bradshaw JL, Galluci RM (1998) Response (re-) programming in aging: a kinematic analysis. J Gerontol Med Sci 53:M222-M227

8. Blaszczyk JW, Orawiec R, Duda-Kiodowska D, Opala G (2007) Assessment of postural instability in patients with Parkinson's disease. Exp Brain Res 183:107-114 doi:10.1007/s00221-0071024-y

9. Bohnen NI, Albin RL, Koeppe RA, Wernette KA, Kilbourn MR, Minoshima S et al (2006) Positron emission tomography of monoaminergic vesicular binding in aging and Parkinson disease. J Cereb Blood Flow Metab 26:1198-1212

10. Braak H, Bohl JR, Müller CM, Rüb U, de Vos RAI, Del Tredici K (2006) Stanley Fahn lecture 2005: the staging procedure for the inclusion body pathology associated with Sporadic Parkinson's disease reconsidered. Mov Disord 21:2042-2051 doi:10.1002/ mds. 21065

11. Braak H, de Vos RAI, Jansen ENH, Bratzke H, Braak E (1998) Neuropathological hallmarks of Alzheimer's and Parkinson's diseases. Prog Brain Res 117:267-285 doi:10.1016/S0079-6123 (08)64021-2

12. Brauer LH, Cramblett MJ, Paxton DA, Rose JE (2001) Haloperidol reduces smoking of both nicotine-containing and denicotinized cigarettes. Psychopharmacology 159:31-37 doi:10.1007/ s002130100894

13. Brebion G (2001) Language processing, slowing, and speed/ accuracy trade-off in the elderly. Exp Aging Res 27:137-150 doi:10.1080/036107301750073999

14. Brebion G (2003) Working memory, language comprehension, and aging: Four experiments to understand the deficit. Exp Aging Res 29:269-301 doi:10.1080/03610730303725

15. Brebion G, Smith MJ, Ehrlich M-F (1997) Working memory and aging: deficit or strategy differences. Aging Neuropsychol Cogn 4:58-73 doi:10.1080/13825589708256636

16. Chaco J, Abramsky O (2004) The effect of L-dopa on postural reflexes in Parkinson's disease. J Neurol 2:122-125

17. Cham R, Studenski SA, Perera S, Bohnen NI (2008) Striatal dopaminergic denervation and gait in healthy adults. Exp Brain Res 185:391-398 doi:10.1007/s00221-007-1161-3

18. Chong RKY, Jones CL, Horak FB (1999) Postural set for balance control is normal in Alzheimer's but not in Parkinson's disease. J Gerontol Biol Sci Med Sci 54A:M129-M135

19. Diamond BJ, DeLuca J, Rosenthal D, Vlad R, Davis K, Lucas G et al (2000) Information processing in older versus younger adults: Accuracy versus speed. Int J Rehabil Health 5:55-64 doi:10.1023/A:1012911203468

20. Diener HC, Dichgans J (1992) Pathophysiology of cerebellar ataxia. Mov Disord 7:95-109 doi:10.1002/mds.870070202

21. Forno LS (1990) Pathology of Parkinson's disease: the importance of the substantia nigra and Lewy bodies. In: Stern GM (ed) Parkinson's disease. Chapman \& Hall, London, pp 185-238

22. Fredericks CM (1996) Disorders of the cerebellum and its connections. In: Fredericks CM, Salakin LK (eds) Pathophysiology of the motor systems. FA Davis, Philadelphia, pp 445-466

23. Friston KJ, Buechel C, Fink GR, Morris J, Rolls E, Dolan RJ (1997) Psychophysiological and modulatory interactions in neuroimaging. Neuroimage 6:218-229 doi:10.1006/nimg.1997.0291

24. Gao K, Calabrese JR (2005) Newer treatment studies for bipolar depression. Bipolar Disord 7:13-23 doi:10.1111/j.1399618.2005.00250.x

25. Goldberg A, Hernandez ME, Alexander NB (2005) Trunk repositioning errors are increased in balance-impaired older adults. J Gerontol Biol Sci Med Sci 60A:1310-1314

26. Gopher D, Sanders AF (1984) S-Oh-R: Oh stages! Oh resources!. In: Prinz V, Sanders AF (eds) Cognition and motor processes. Springer, Berlin, pp 231-253
27. Granacher U, Gollhofer A, Strass D (2006) Training induced adaptations in characteristics of postural reflexes in elderly men. Gait Posture 24:459-466

28. Gurney K, Prescott T, Wickens JR, Redgrave P (2004) Computational models of the basal ganglia: from robots to membranes. Trends Neurosci 27:453-459 doi:10.1016/j.tins.2004.06.003

29. Halliday G, Hely M, Reid W, Morris J (2008) The progression of pathology in longitudinally followed patients with Parkinson's disease. Acta Neuropathol 115:409-415 doi:10.1007/s00401-0080344-8

30. Hasher L, Zacks RT (1979) Automatic and effortful processes in memory. J Exp Psychol Gen 108:356-388 doi:10.1037/00963445.108.3.356

31. Hertzog C, Vernon MC, Rypma B (1993) Age differences in mental rotation task performance: the influence of speed/accuracy tradeoffs. J Gerontol Psychol Sci 48:150-156

32. Heuninckx S, Wenderoth N, Debaere P, Peeters R, Swinnen SP (2005) Neural basis of aging: the penetration of cognition into action control. J Neurosci 25:6787-6796 doi:10.1523/JNEUROSCI.1263-05.2005

33. Heuninckx S, Wenderoth N, Swinnen SP (2008) Systems neuroplasticity in the aging brain: Recruiting additional neural resources for successful motor performance in elderly persons. J Neurosci 28:91-99 doi:10.1523/JNEUROSCI.3300-07.2008

34. Horak FB, Shupert CL, Mirka A (1989) Components of postural dyscontrol in the elderly: A review. Neurobiol Aging 10:727 doi:10.1016/0197-4580(89)90010-9

35. Hornykiewicz O (1983) Parkinson's disease and the aging basal ganglia. In: Gispe WH, Traber J (eds) Aging of the brain. vol. 7. Elsevier, Amsterdam, pp 253-274

36. Hutchinson S, Kobayashi M, Horkan CM, Pascual-Leone A, Alexander MP, Schlaug G (2002) Age-related differences in movement representation. Neuroimage 17:1720-1728 doi:10.1006/nimg.2002.1309

37. Kagerer S, Winter C, Moller HJ, Soyka M (2003) Effects of haloperidol and atypical neuroleptics on psychomotor performance and driving ability in schizophrenic patients. Results from an experimental study. Neuropsychobiol 47:212-218 doi:10.1159/ 000071217

38. Kemoun G, Defebvre L, Watelain E, Guieu JD, Destee A (2003) Parkinson's disease as a model of aging: prospective analysis of gait disorders. Rev Neurol 159:1028-1037

39. Langenecker SA, Briceno EM, Hamid NM, Nielson KA (2007) An evaluation of distinct volumetric and functional MRI contributions toward understanding age and task performance: a study in the basal ganglia. Brain Res 1135:58-68 doi:10.1016/j.brainres.2006. 11.068

40. Logan GD (1978) Attention in character-classification tasks: evidence for the automaticity of component stages. J Exp Psychol Gen 107:32-63 doi:10.1037/0096-3445.107.1.32

41. Marsden CD (1985) Defects of movement in Parkinson's disease. In: Delwaide PJ, Agnoli A (eds) Clinical neurophysiology in Parkinsonism. Elsevier, Amsterdam, pp 107-115

42. Marsden CD (1990) Neurophysiology. In: Stern G (ed) Parkinson's disease. 2nd edn. Chapman \& Hall, New York, pp 57-98

43. Maschke M, Tuite PJ, Pickett K, Wächter T, Konczak J (2005) The effect of subthalamic nucleus stimulation on kinaesthesia in Parkinson's disease. J Neurol Neurosurg Psychiatry 76:569-571 doi:10.1136/jnnp.2004.047324

44. Massion J (1998) Postural control systems in developmental perspective. Neurosci Biobehav Rev 22:465-472 doi:10.1016/ S0149-7634(97)00031-6

45. Melzer I, Benjuya N, Kaplanski J (2001) Age-related changes of postural control: effect of cognitive tasks. Gerontol 47:189-194 doi: $10.1159 / 000052797$ 
46. Metter EJ (1992) Brain glucose metabolism in subcortical dementias. In: Vallar G, Cappa SF, Wallesch CW (eds) Neuropsychological disorders associated with subcortical lessions. Oxford University Press, Oxford, pp 242-259

47. Morgan M, Phillips JG, Bradshaw JL, Mattingley JB, Iansek R, Bradshaw JA (1994) Age-related motor slowness: simply strategic. J Gerontol Med Sci 49:M133-M139

48. Norman DA, Bobrow DG (1975) On data-limited and resourcelimited processes. Cognit Psychol 7:44-64 doi:10.1016/00100285(75)90004-3

49. Parker D, Macdonald L, Sutcliffe P, Rabbitt P (2001) Confidence and the older driver. Ageing Soc 21:169-182 doi:10.1017/ S0144686X01008133

50. Perrin PP, Jeandel C, Perrin CA, Bene MC (1997) Influence of visual control, conduction and central integration on static and dynamic balance in healthy older adults. Gerontol 43:223-231

51. Phillips JG, Martin KE, Bradshaw JL, Iansek R (1994) Could bradykinesia in Parkinson's disease simply be compensation. J Neurol 241:439-447 doi:10.1007/BF00900963

52. Phillips JG, Müller F, Stelmach GE (1989) Movement disorders and the neural basis of motor control. In: Wallace SA (ed) Perspectives on the coordination of movement. Elsevier, NorthHolland, pp 367-413

53. Phillips JG, Schiffter T, Nicholls Bradshaw MEJL, Iansek R, Saling LL (1999) Does old age or Parkinson's disease cause bradyphrenia. J Gerontol Biol Sci Med Sci 54:M404-M409

54. Phillips JG, Triggs TJ (2001) Whither automaticity and human performance. In: Columbus F (ed) Advances in psychology research. vol. 4. Nova Science, Happauge, NY, pp 151-173

55. Pier MPBI, Hulstijn W, Sabbe BGC (2004) Psychomotor retardation in elderly depressed patients. J Affect Disord 81:7377 doi:10.1016/j.jad.2003.08.002

56. Rabbitt P, Scott M, Thacker N, Lowe C, Jackson A, Horan M et al (2006) Losses in gross brain volume and cerebral blood flow account for age-related differences in speed but not in fluid intelligence. Neuropsychol 20:549-557 doi:10.1037/08944105.20.5.549

57. Rammsayer TH, Rodewald S, Groh D (2000) Dopamine-antagonistic, anticholinergic, and GABAergic effects on declarative and procedural memory functions. Brain Res Cogn Brain Res 9:61-71 doi:10.1016/S0926-6410(99)00045-2

58. Rammsayer T, Stahl J (2006) Sensorimotor effects of pergolide, a dopamine agonist, in healthy subjects: a lateralized readiness potential study. Psychopharmacol 187:36-46

59. Rubin DC (1999) Frontal-striatal circuits in cognitive aging: evidence for caudate involvement. Aging Neuropsychol Cogn 6:241-259 doi:10.1076/1382-5585(199912)06:04;1-B;FT241

60. Saling LL, Phillips JG (2002) Age-related changes in the kinematics of curved drawing movements: relationship between tangential velocity and the radius of curvature. Exp Aging Res 28:215-229 doi:10.1080/03610730252800210

61. Saling LL, Phillips JG (2005) Variations in the relationship between radius of curvature and velocity as a function of joint motion.. Hum Mov Sci 224:731-743 doi:10.1016/j.humov.2005.09.001

62. Saling LL, Phillips JG (2007) Automaticity: efficient not mindless. Brain Res Bull 73:1-20 doi:10.1016/j.brainresbull.2007.02.009

63. Salthouse TA (1985) Speed of behavior and its implications for cognition. In: Birren JE, Schaie KW (eds) Handbook of the psychology of aging. 2nd edn. Reinhold, New York, pp 400-426

64. Samuels ER, Hou RH, Langley RW, Szabadi E, Bradshaw CM (2006) Comparison of pramipexole and amisulpride on alertness, autonomic and endocrine functions in healthy volunteers. Psychopharmacol 187:498-510 doi:10.1007/s00213-006-0443-y
65. Sawabini KA, Watts RL (2004) Treatment of depression in Parkinson's disease. Parkinsonism Relat Disord 10:S37-S41 doi:10.1016/j.parkreldis.2004.02.002

66. Seiss E, Praamstra P (2004) The basal ganglia and inhibitory mechanisms in response selection: Evidence from subliminal priming of motor responses in Parkinson's disease. Brain 127:330-339 doi:10.1093/brain/awh043

67. Stelmach GE, Phillips JG (1992) Motor control in Parkinson's disease. In: Turnbull GI (ed) Physical therapy management of Parkinson's disease. Livingstone, Edinburgh, pp 37-48

68. Stelmach GE, Phillips JG, DiFabio RP, Teasdale N (1989) Age, functional postural reflexes, and voluntary sway. J Gerontol Biol Sci 44:B100-B106

69. Takahashi H, Yoshida K, Higuchi H, Shimizu T, Inoue T, Koyama T (2003) Addition of a dopamine agonist, cabergoline, to a serotoninnoradrenalin reuptake inhibitor, milnacipran as a therapeutic option in the treatment of tefractory depression: Two case reports. Clin Neuropharmacol 26:230-232 doi:10.1097/00002826-20030900000005

70. Taniwaki T, Okayama A, Yoshiura T, Togao O, Nakamura Y, Yamasaki T et al (2007) Age-related alterations of the functional interactions within the basal ganglia and cerebellar motor loops in vivo. Neuroimage 36:1263-1276 doi:10.1016/j.neuro image.2007.04.027

71. Touron DR, Hertzog C (2004) Strategy shift affordance and strategy choice in young and older adults. Mem Cognit 32:298-310

72. Ustinova KI, Ioffe ME, Chernikova LA (1999) Age-related features of the voluntary control of the upright posture. Hum Physiol 29:724-728 doi:10.1023/B:HUMP.0000008845.17747.e8

73. Vickers D, Burt J, Smith P, Brown M (1985) Experimental paradigms emphasising state or process limitations: I Effects on speed-accuracy tradeoffs. Acta Psychol (Amst) 59:129-161 doi:10.1016/0001-6918(85)90017-4

74. Volkow ND, Logan J, Fowler JS, Wang GJ, Gur RC, Wong C et al (2000) Association between age-related decline in brain dopamine activity and impairment in frontal and cingulate metabolism. Am J Psychiatry 157:75-80 doi:10.1176/appi.ajp.157.10.1709

75. Welford AT (1980) Relationships between reaction time and fatigue, stress age and sex. In: Welford AT (ed) Reaction times. Academic, London, pp 321-354

76. Wichmann T, DeLong MR (2003) Pathophysiology of Parkinson's disease: The MPTP primate model of the human disorder. In: Federoff HJ, Burke RE, Fahn S, Fiskum G (eds) Parkinson's disease: The life cycle of the dopamine neuron. New York Academy of Sciences, New York, pp 199-213

77. Wu T, Hallett M (2005a) A functional MRI study of automatic movements in patients with Parkinson's disease. Brain 128:2250 2259 doi:10.1093/brain/awh569

78. Wu T, Hallett M (2005b) The influence of normal human ageing on automatic movements. J Physiol 562:605-615 doi:10.1113/ jphysiol.2004.076042

79. Wu T, Kansaku K, Hallet M (2004) How self-initiated memorized movements become automatic: a functional MRI study. J Neurophysiol 91:1690-1698 doi:10.1152/jn.01052.2003

80. Zack M, Poulos CX (2007) A D2 antagonist enhances the rewarding and priming effects of a gambling episode in pathological gamblers. Neuropsychopharmacol 32:1678-1686 doi:10.1038/sj.npp.1301295

81. Zia S, Cody F, O'Boyle D (2000) Joint position sense is impaired by Parkinson's disease. Ann Neurol 47:218-228 doi:10.1002/ 1531-8249(200002)47:2<218::AID-ANA12>3.0.CO;2-\#

82. Zirnheld PJ, Carroll CA, Kieffaber PD, O’Donnell BF, Shekhar A, Hetrick WP (2004) Haloperidol impairs learning and error-related negativity in humans. J Cognitive Neurosci 16:1098-1112 